\title{
PUENTE SOBRE EL RÍO GUADALETE DE TABLERO DE VIGAS PRETENSADAS PREFABRICADAS DE HORMIGÓN DE ALTA RESISTENCIA
}

\author{
(BRIDGE ACROSS GUADALETE RIVER BUILT OF PREFABRICATED BEAMS OF PRESTRESSED \\ HIGH STRENGTH CONCRETE)
}

Fernando Hue García, Ingeniero de Caminos. DRAGADOS

Guillermo Ontañón Carrera, Ingeniero de Caminos. INTECSA

Jesús Rodriguez Santiago, Dr. Ingeniero de Caminos. GEOCISA

ESPAÑA

Fecha de recepción: 21-II-97

$562-152$

\section{RESUMEN}

Se describe el proyecto y la construcción de un puente sobre el río Guadalete, cuya realización y cálculo se basan en el empleo de vigas pretensadas prefabricadas, concretamente de Hormigón de Alta Resistencia (HAR).

Partiendo de diversas soluciones de proyecto, se van definiéndo aspectos tales como la cimentación, pilas y estribos, vigas, losas, etc. Se continúa con la prefabricación, construcción y cálculo, asi como los ensayos realizados sobre el hormigón y las vigas de dicha obra.
SUMMARY

Project and construction of a bridge across Guadalete river which construction project is based on the use of prefabricated prestressed beams of High Strength Concrete (HSC).

Starting from the different project solutions, the description develops aspects such as foundations, piles, butresses, beams, slabs, etc., and continues with prefabrication, calculus and construction. It also describes the tests carried out on the beams, and the concrete that has been used in this work.

\section{Introducción}

El puente sobre el río Guadalete, estructura E-5 de la variante de El Puerto de Santa María en la provincia de Cádiz es el primer puente que ha sido proyectado, calculado y construido en España con un tablero formado con vigas prefabricadas pretensadas de Hormigón de Alta Resistencia (HAR).

Esta variante de la carretera radial N-IV e itinerario europeo E-05 tiene características de autovía. El puente está diseñado con dos calzadas independientes de $11,50 \mathrm{~m}$ de anchura total cada una, separadas $2 \mathrm{~m}$ entre ellas y con $210 \mathrm{~m}$ de longitud total. Sirve para pasar sobre el río
Guadalete, muy cerca de su desembocadura en el océano Atlántico.

\section{Soluciones estudiadas}

En el proyecto original de la autovía, el puente estaba formado por siete vanos de $30 \mathrm{~m}$ de longitud en cada calzada. Cada tablero tenía cinco vigas prefabricadas pretensadas de hormigón $\mathrm{H}-45,1,53 \mathrm{~m}$ de canto, separadas 2,40 $\mathrm{m}$ entre ejes, unidas por una losa de tablero ejecutada in situ con hormigón $\mathrm{H}-25$, de $0,20 \mathrm{~m}$ đe espesor.

El puente está situado muy cerca de la desembocadura de los ríos Guadalete y San Pedro. Esta zona es un terreno de 
marisma, con una capa superior de arcillas cuaternarias muy blandas, situada sobre estratos terciarios de arenas densas y gravas y arcillas duras. Este terreno obligaba a emplear en el puente una cimentación mediante pilotes, que alcanzasen una profundidad de unos $19 \mathrm{~m}$ bajo el nivel del suelo, para tener suficiente capacidad portante.

Debido a este terreno, las doce pilas y los cuatro estribos estaban soportados por tres pilotes cilíndricos huecos pretensados, tipo Raymond, de 54" de diámetro, prefabricados e hincados en el terreno con grúa y martillo de aire comprimido tipo Vulcan y unidos, en su parte superior, por cabeceros prefabricados de hormigón armado.
Este tipo de cimentación es caro y se pensó en reducir el peso del puente para disminuir el número necesario de pilotes. Para ello había que resolver los tableros con un menor número de vigas, empleando en ellas un hormigón de mayor resistencia, como el HAR.

La utilización de este tipo de material tenía una ventaja añadida. Al HAR también se le denomina Hormigón de Altas Prestaciones por sus mejores cualidades; en general, por su mayor durabilidad, por su permeabilidad al ion $\mathrm{Cl}^{-}$, muy inferior y, por tanto, su mayor protección del acero de armar y pretensar frente a la corrosión.
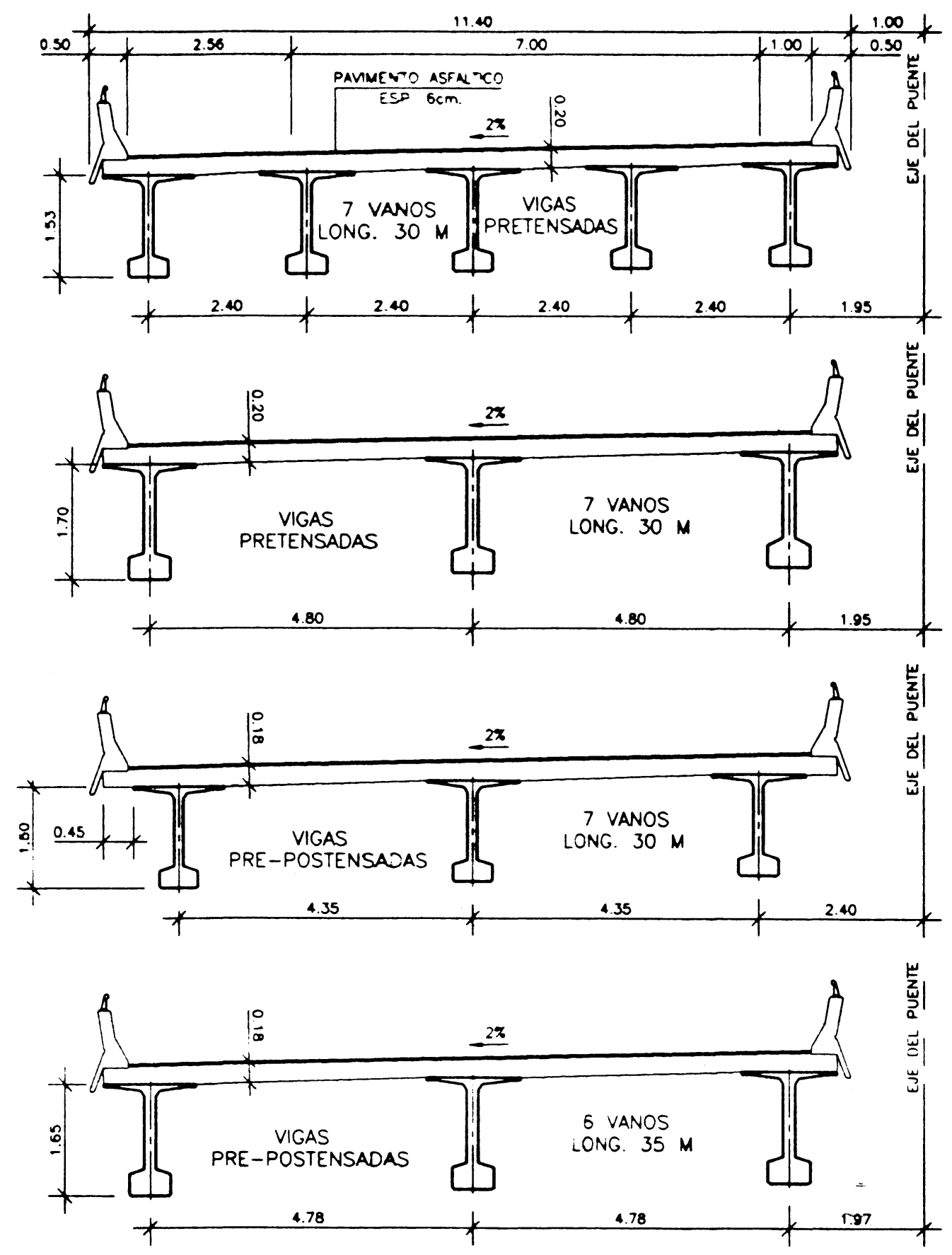

Fig. 1.- Solución original con $H-45$ y soluciones $A, B$ y C con $H A R H-80$. 
Al estar el puente muy cerca del océano Atlántico, recibe de él vientos húmedos y salinos, que lo colocan en una atmósfera muy agresiva, empeorada por la reducida distancia, sólo unos $3 \mathrm{~m}$, entre la cara inferior de las vigas y el nivel del río, que oscila con las mareas.

La construcción de este puente con vigas de HAR permitía, además, conseguir experiencia en una obra real, de su empleo en elementos prefabricados y comparar su comportamiento, a lo largo del tiempo, con vigas de hormigón de calidad, pero no HAR, utilizadas en puentes próximos de la misma obra de la variante.
A partir de la solución de proyecto, con la idea de reducir el peso del tablero y optimizar el coste total del puente, tableros, estribos y pilas con sus pilotes, se estudiaron varias soluciones, utilizando hormigón $\mathrm{H}-80$ en las vigas prefabricadas, colocando estas vigas, bien al borde del tablero, bien más juntas, para reducir esfuerzos sobre ellas.

Las soluciones estudiadas para los tableros fueron las descritas a continuación (Figs. 1 y 2):

Solución A: Siete vanos de $30 \mathrm{~m}$ de longitud por calzada, con tres vigas pretensadas de $1,70 \mathrm{~m}$ de canto, colocadas al
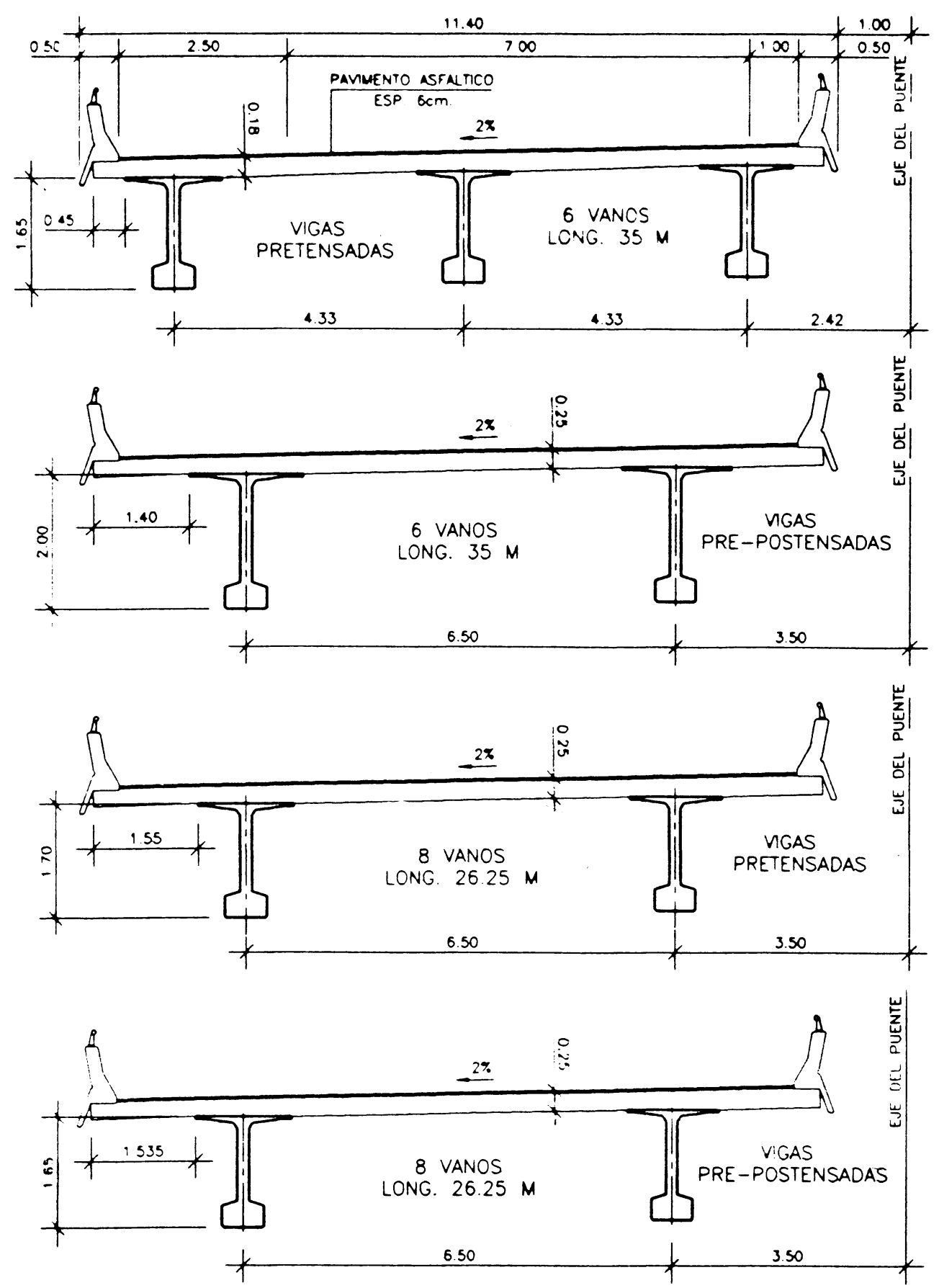

Fig. 2.- Soluciones D, E. Fy G con HAR H-80. 
borde de un tablero de $20 \mathrm{~cm}$ de espesor. En esta solución no hay tendones de postensado y, por lo tanto, tampoco anclajes que sobresalgan por encima de la cara superior de las vigas. Además, al ir colocadas en el borde del tablero, no se necesita encofrado en el voladizo, que es dificil y costoso de disponer.

Solución B: Siete vanos de $30 \mathrm{~m}$ de longitud por calzada, con tres vigas pre-postensadas de $1,50 \mathrm{~m}$ de canto, colocadas dejando un vuelo de $45 \mathrm{~cm}$ en el borde de un tablero de $18 \mathrm{~cm}$ de espesor. En esta solución, sí hay tendones de postensadoy, por lo tanto, también anclajes que sobresalen por encima de la cara superior de las vigas. Además, se necesita encofrado en el vuelo, pero el menor canto del tablero permite reducir el coste de los terraplenes de acceso.

Solución C: Seis vanos de $35 \mathrm{~m}$ de longitud, con tres vigas pre-postensadas de $1,65 \mathrm{~m}$ de canto, al borde de un tablero de $18 \mathrm{~cm}$ de espesor.

Solución D: Seis vanos de $35 \mathrm{~m}$ de longitud, con tres vigas pretensadas de 1,65 de canto, colocadas a $45 \mathrm{~cm}$ del borde de un tablero de $18 \mathrm{~cm}$ de espesor.

Solución E: Seis vanos de $35 \mathrm{~m}$ de longitud, con dos vigas pre-postensadas de $2 \mathrm{~m}$ de canto, colocadas a $1,40 \mathrm{~m}$ del borde de un tablero de $25 \mathrm{~cm}$ de espesor.
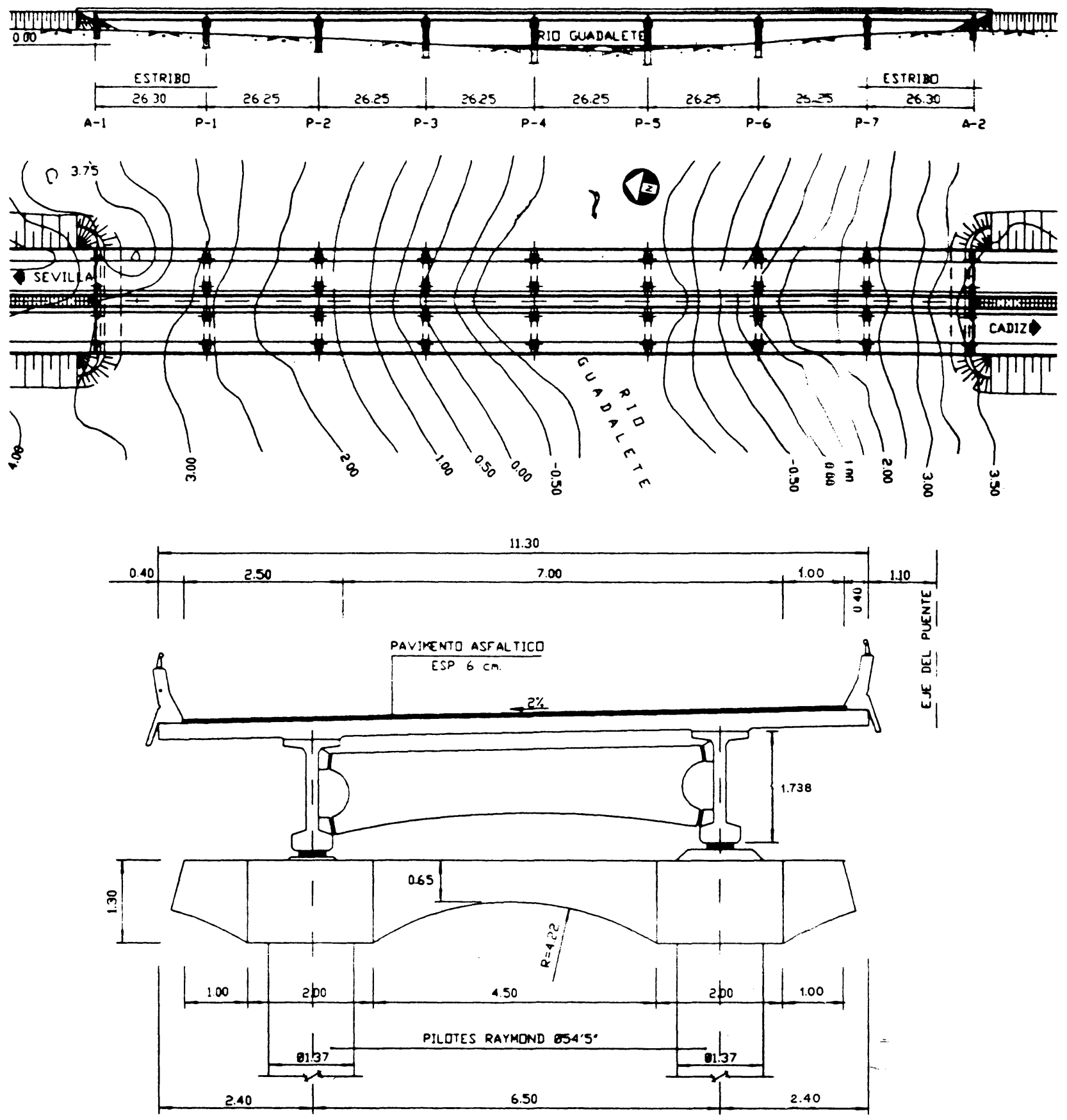

Fig. 3.- Alzado, planta y sección transversal del puente. 
Todas estas soluciones necesitan que las pilas y los estribos estén soportados por tres pilotes cada uno.

Solución F: Ocho vanos de 26,25 m de longitud, con dos vigas pretensadas de $1,70 \mathrm{~m}$ de canto, colocadas a $1,55 \mathrm{~m}$ del borde de un tablero de $25 \mathrm{~cm}$ de espesor. Esta solución tiene más vanos, pero sólo necesita ser cimentada mediante dos pilotes por pila o estribo, reduciéndose el número total de pilotes y el coste de la cimentación.

Solución G: Ocho vanos de 26,25 m de longitud, con dos vigas pre-postensadas de $1,65 \mathrm{~m}$ de canto, colocadas a $1,535 \mathrm{~m}$ del borde de un tablero de $25 \mathrm{~cm}$ de espesor. Esta solución es muy similar a la anterior $\mathrm{F}$, pero algo más cara, debido al postensado.

Se eligió la solución $\mathrm{F}$, la cual se modificóligeramente para incrementar el espesor de la cabeza superior de la viga, aumentando su canto hasta $1,738 \mathrm{~m}$ y reduciendo su anchura de $1,40 \mathrm{~m}$ a $0,90 \mathrm{~m}$, de forma que se conservase el mismo área de la cabeza superior de la viga.

Estas vigas necesitaban ser pretensadas también en la cabeza superior.

\section{Descripción del puente (Fig. 3)}

\subsection{Cimentación}

La cimentación de cada pila o estribo está formada por dos pilotes circulares huecos, tipo Raymond, de 54" de diámetro exterior y 5 " de espesor de pared, prefabricados y pretensados, colocados a una distancia de 6,50 m entre ejes.

El hormigón utilizado es de tipo H-45. Los pilotes están postensados mediante 18 cables de 0,6 " de diámetro, superestabilizados, tipo ASTM A-416 y Grado 270, con una carga de rotura de $261 \mathrm{kN}$. Estos cables se tensan a $196 \mathrm{kN}$.

Con este diseño de puente, los pilotes tienen agotada, casi totalmente, su capacidad resistente para las cargas permanentes y sobrecargas de cálculo, por lo que noexiste ningún sobrecoste, debido a la necesidad de utilizar un número entero de pilotes en cada pila o estribo.

\subsection{Pilas y estribos}

Cada pila está formada por un cabecero de hormigón armado prefabricado, de canto variable, entre $0,65 \mathrm{~m} \mathrm{y}$ $1,30 \mathrm{~m}$, y 1,40 m de anchura, que se coloca en la parte superior de dos pilotes. En la zona de unión la anchura es de $2 \mathrm{~m}$.

Las juntas de unión entre pilotes y cabeceros de pilas se llevan a cabo mediante una conexión de $3 \mathrm{~m}$ de longitud, en el interior de la parte superior del pilote, hormigonada in situ, con armaduras de unión entre pilote y cabecero.
Cada estribo está formado por un cabecero de hormigón armado hecho in situ, de $1,30 \mathrm{~m}$ de canto y $1,40 \mathrm{~m}$ de anchura, colocado en la parte superior de dos pilotes. El espaldón es de 0,40 $\mathrm{m}$ de espesor. El estribo lleva aletas para la contención del terraplén.

La conexión entre pilotes y cabeceros en los estribos, es similar a la descrita para las pilas y se hormigonan simultáneamente con el cabecero.

La distancia entre pilotes en pilas y cabeceros coincide con el intereje entre vigas de tablero, de forma que las reacciones verticales de los tableros pasan directamente a los pilotes, sin producir flexiones en los cabeceros. Los cabeceros de pilas y estribos solamente trabajan a flexión frente a los esfuerzos transversales de viento y sismo, rigidizando el puente en ese sentido.

El hormigón utilizado en los cabeceros de pilas y estribos es de tipo H-35, armado con barras de acero corrugado tipo AEH-500.

\subsection{Vigas de tablero}

Cada tablero tiene dos vigas prefabricadas de $1,738 \mathrm{~m}$ de canto y 26,15 m de longitud (Fig. 4), situadas a una distancia de 6,50 m entre sus ejes. $\mathrm{El}$ espesor del alma es

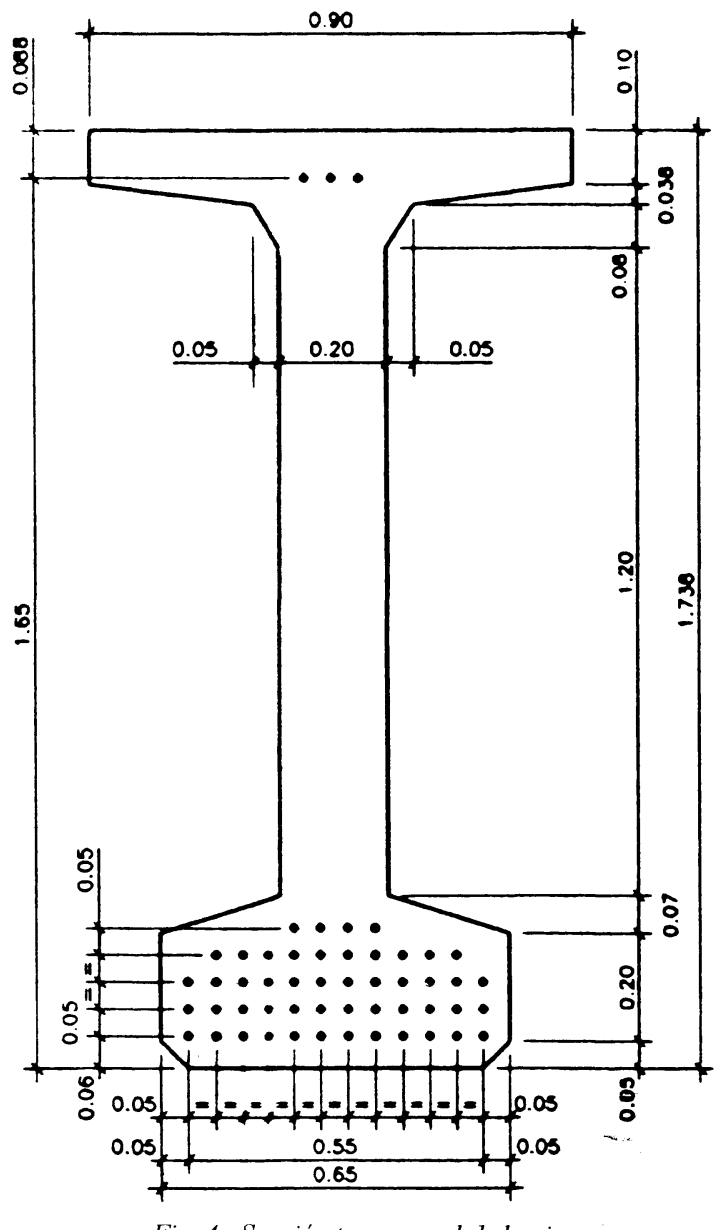

Fig. 4.- Sección transversal de la viga. 
$20 \mathrm{~cm}$, la anchura de la cabeza superior es de $90 \mathrm{~cm}$ y la anchura de la inferior es de $65 \mathrm{~cm}$.

El hormigón utilizado es HAR de tipo H-80. Estas vigas están pretensadas con 50 cables en la cabeza inferior y 3 en la superior, de 0,6" de diámetro, del mismo tipo utilizado en los pilotes y descrito anteriormente.

\section{4 Losas de tablero}

La losa de cada tablero tiene $26 \mathrm{~cm}$ de espesor y 11,30 de anchura. Se prefabrica en once piezas de toda la anchura y 2,36 m de longitud de tablero. Estas piezas se unen entre sí por juntas transversales al tablero de $52 \mathrm{~cm}$ de anchura y con las vigas mediante aberturas de 1,05 x 0,60 m, donde se concentran las armaduras de conexión con las vigas. El hormigón utilizado es de tipo H-35, armado con barras de acero corrugado tipo AEH-500

\section{Prefabricación del puente}

Este puente ha sido prefabricado casi en su totalidad, solamente se han construido in situ los estribos y las juntas de unión entre pilotes y cabeceros de pilas, entre vigas y losas de tablero y entre estas losas.

Para una superficie de puente de $4.750 \mathrm{~m}^{2}$ construidos, se han hormigonado in situ $36 \mathrm{~m}^{3}$ en las juntas de las pilas y $234 \mathrm{~m}^{3}$ en las juntas de las losas de tablero, aparte de los estribos.
El cabecero de éstos, también podría haber sido prefabricado, pero la necesidad de ejecutar in situ los espaldones y las aletas y la reducida repetición de piezas lo desaconsejó.

Los pilotes se prefabrican en secciones de $5 \mathrm{~m}$ de longitud típica, mediante una máquina que las centrifuga, consiguiéndose una compacidad muy elevada en el hormigón. El curado de secciones se realiza con vapor calentado. Las secciones llevan unos orificios longitudinales donde se colocan los cables de pretensado, que sirven para comprimir el pilote formado con ellas y unirlas entre sí.

En las caras de unión se extiende una capa de resina epoxi para pegadoy para un buen asiento. Los cables de pretensado se tensan contra unos anclajes temporales, que se retiran una vez que ha endurecido suficientemente el mortero inyectado en los orificios de los cables. El anclaje de éstos pasa entonces a ser por adherencia. Los pilotes se prefabricaron en longitudes de $25 \mathrm{~m}$.

Los cabeceros y las losas se fabricaron en encofrados fijos apoyados en el suelo.

Las vigas se prefabrican en un encofrado de $110 \mathrm{~m}$ de largo, montado sobre una bancada con dispositivos de vibración de forma que durante el hormigonado vibra todo el encofrado. Las vigas se hormigonaron en ocho grupos de cuatro vigas (Fig. 5).

Las jaulas de armadura pasiva de las vigas, con los cables de pretensado dentro de ellas y los tapes finales de cada una,

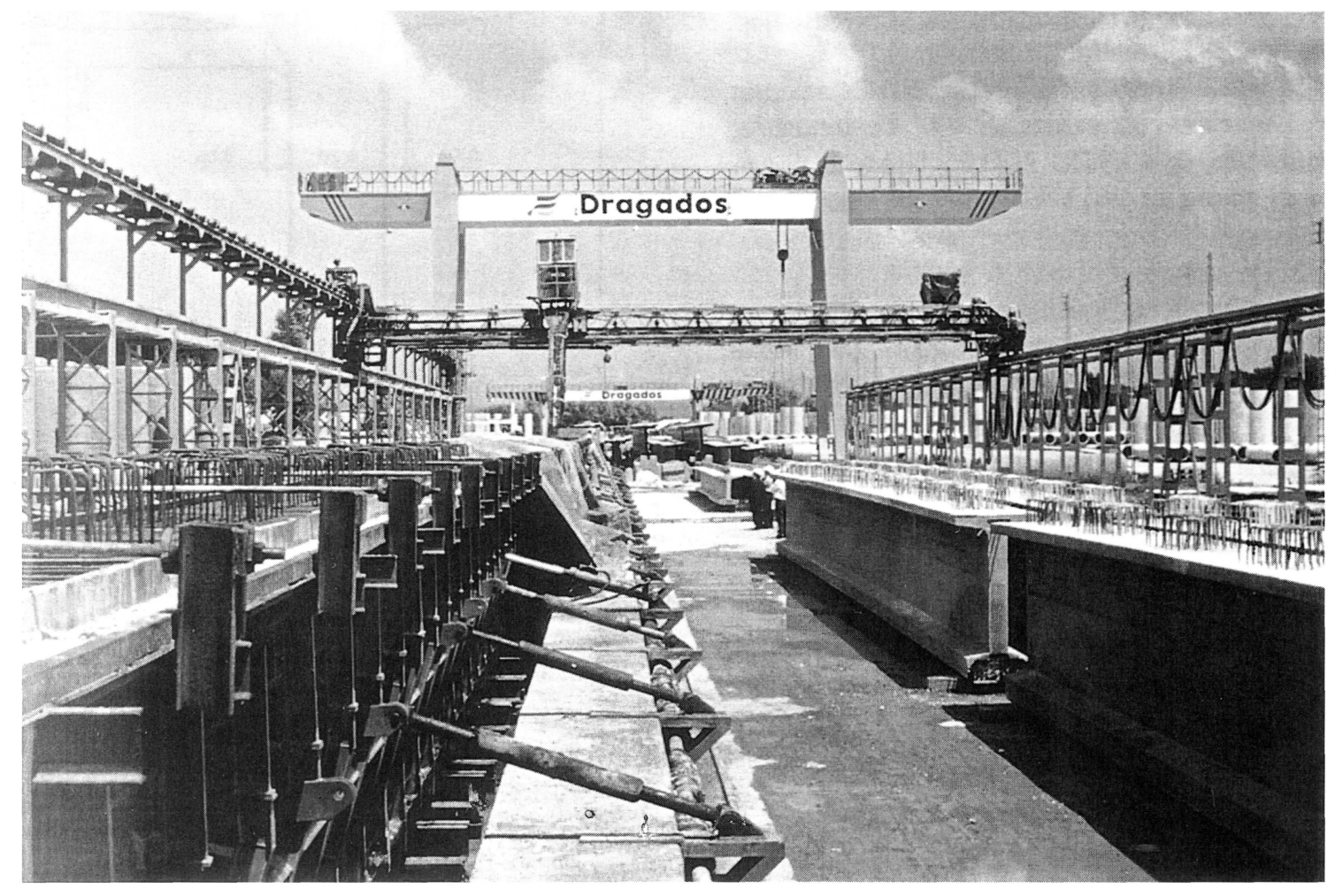


se colocan dentro del encofrado suspendidas de unos pórticos, que se desplazan conjuntamente sobre unos carriles situados a ambos lados de la bancada, cerrándose después, girando los paneles laterales.

Los cables de pretensado colocados a lo largo de todo el encofrado, al ser tensados, se anclan temporalmente con casquillos de anclaje. Estos casquillos apoyan sobre unas vigas metálicas horizontales, apoyadas, a su vez, sobre los extremos de la bancada. Esta bancada soporta por compresión las fuerzas del pretensado.

El hormigón se lleva al encofrado mediante cintas transportadoras y un carro repartidor, dispuesto como un pórtico-grúa. Después del hormigonado de cada viga, se cubre su zona de encofrado con lonas y se inyecta vapor a $50^{\circ} \mathrm{C}$ durante doce horas, empezando dos horas después del hormigonado.

Después del curado a vapor se comprueba, con probetas curadas en la misma bancada, que el hormigón de las vigas tiene la resistencia que se requiere en el proyecto, para transferir las fuerzas del pretensado, procediéndose al corte de cables cuando se ha alcanzado dicha resistencia.

Después del corte de los cables, se abre el encofrado girando los paños laterales y se sacan las vigas, con uno o dos puentes grúa, según su peso, y se llevan a la zona de acopio, donde se continúa el curado por aspersión de agua.

Los pilotes y las vigas se prefabricaron en una factoría de piezas prefabricadas, que es propiedad de Dragados y Construcciones, S.A. y está situada muy cerca de la estación de San Roque (Cádiz), a unos $10 \mathrm{~km}$ de Algeciras. Los cabeceros de las pilas y las piezas de las losas de los tableros, se prefabricaron en un taller montado en la propia obra de la variante.

\section{Construcción del puente}

La construcción del puente empezó con la hinca de los 36 pilotes de pilas y estribos. Se utilizó un martillo de simple efecto Vulcan 030 , de $130,8 \mathrm{kN}$ de peso y $119,0 \mathrm{kNm}$ de energía. Los pilotes se transportaron a obra mediante un camión, formado por una cabeza tractora y un dolly o plataforma rodante posterior.

El uso de este tipode pilotes prefabricados de gran diámetro tiene bastantes ventajas:

\section{- Tienen elevadas capacidades de carga}

- El hormigón tiene una gran calidad por su sistema de fabricación. Tiene una elevada resistencia estructural para poder soportar las tensiones durante el proceso de hinca. También tiene una gran compacidad y baja permeabilidad, que le proporcionan una gran resistencia a los ambientes corrosivos, como el marino. Además, el pretensado anula o reduce la fisuración por flexión, ayudando a una mayor durabilidad.

- No tienen, en suelos sueltos o flojos, los problemas de desprendimientos de los pilotes excavados.

- La hinca es un proceso dinámico, que sirve para conocer la capacidad de carga de los pilotes de forma rápida y barata.

Los pilotes, de una longitud total de $25 \mathrm{~m}$, se hincaron en el terreno una longitud de $19,1 \mathrm{~m}$, como promedio.

Para el control de calidad de la hinca de los pilotes se utilizó un sistema analizador PDA (Pile Driving Analyzer), que consiste en colocar en la cabeza del pilote dos acelerómetros y dos transductores de fuerza, que envían sus señales a un ordenador mediante conversores analógicodigitales.

Mediante el programa CAPWAP se analizan las señales, pudiéndose conocer la capacidad portante del pilote, su reparto entre punta y fuste, la distribución de la resistencia a lo largo del fuste y si el pilote está íntegro estructuralmente o tiene secciones fisuradas y dónde las tiene.

Este sistema es mucho más rápido y barato que la prueba de carga estática y por ello se puede realizar en un número mayor de pilotes.

Se comprobaron seis pilotes del puente ( $17 \%)$, haciendo ensayos durante la hinca y la rehinca. Con el fin de conseguir la capacidad portante necesaria para un factor de seguridad de 2,50, hubo que rehincar, siete o más dias después, los pilotes que, según el método de la Ecuación de la Onda, no lo habían alcanzado.

Después de hincados los pilotes, se cortaron a la cota adecuada y se colocaron encima de ellos los cabeceros de las pilas o el encofrado de los estribos, para ser hormigonados estos últimos o las uniones de las pilas.

Las vigas se transportaron a la obra de forma similar a los pilotes. Se colocaron mediante grúa sobre los aparatos de apoyo de neopreno, situados previamente en su posición sobre pilas y estribos.

Las riostras que unen las vigas en las zonas de apoyos, también prefabricadas en obra, y de hormigón armado, se colocaron uniéndolas a las vigas mediante elementos de chapa metálica que se soldaron.

Posteriormente se colocaron las piezas que forman las losas de los tableros, introduciendo por sus aberivias las armaduras de conexión que sobresalían de las caras superiores de las vigas, que estaban agrupadas en las zonas coinciden- 


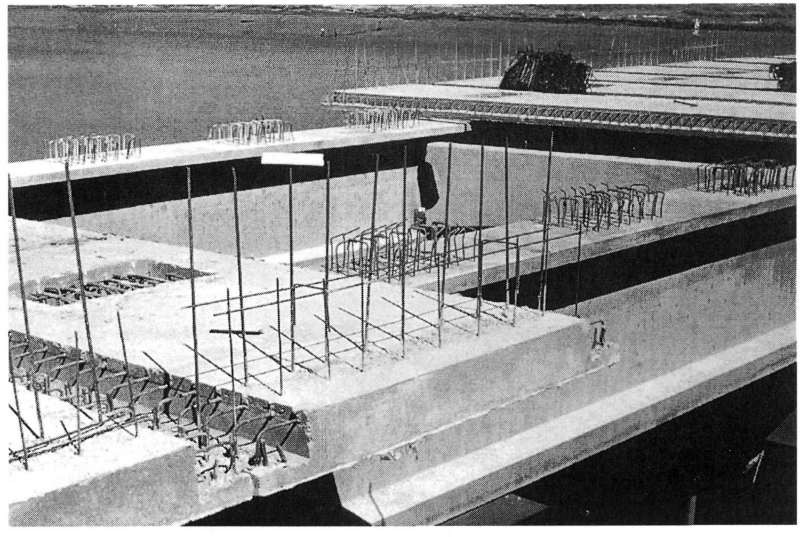

Fig. 6.- Fotografia de las uniones entre losas y vigas en el tablero.

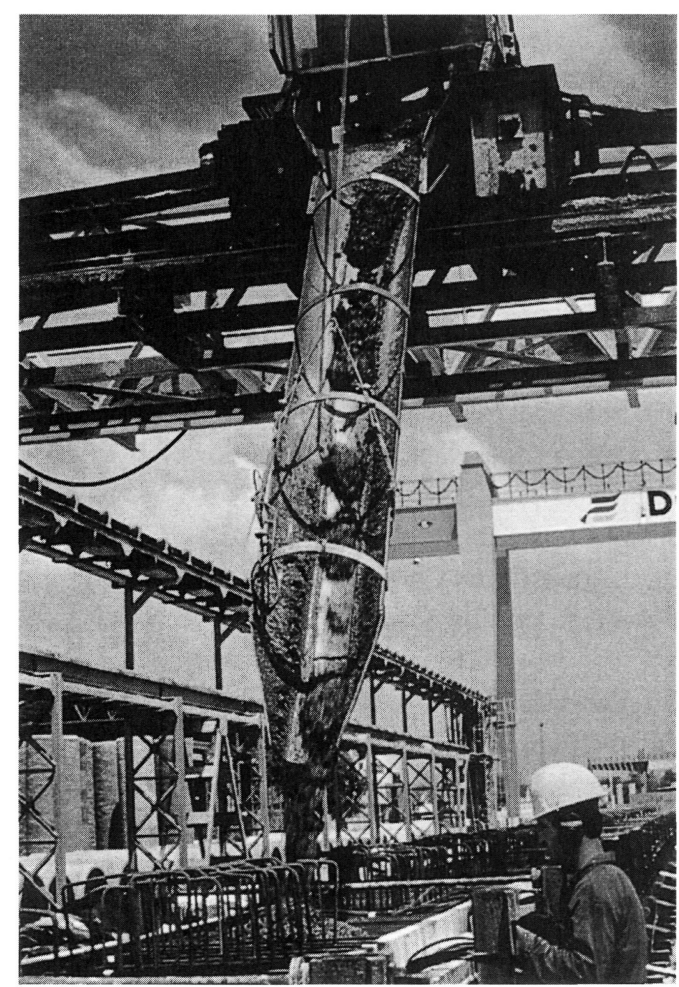

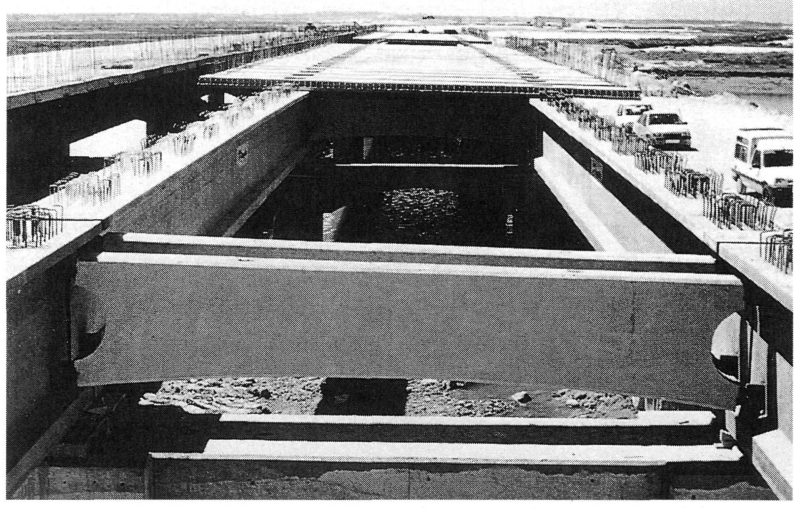

Fig. 7.- Fotografia del puente enfase de montaje de piezas prefabricadas.

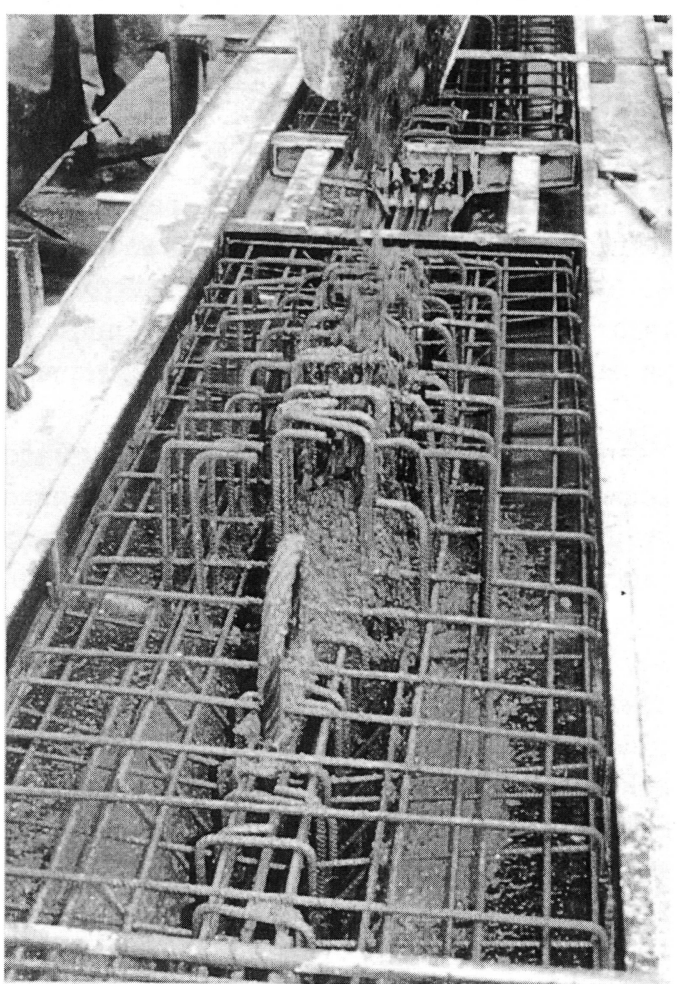

Fig. 8.- Fotografias del proceso de hormigonado de las vigas con $H A R$.

tes con esas aberturas. Con el hormigonado de aberturas y juntas entre piezas, se terminaron los tableros del puente (Figs. 6 y 7 ).

Con la colocación de las defensas laterales de hormigón y el extendido de la capa de rodadura de aglomerado asfáltico, finalizó la construcción del puente.

La prueba de carga estática y dinámica de los tableros dejó el puente en condiciones de ser abierto al tráfico de vehículos.

\section{Hormigón de alta resistencia}

El Hormigón de Alta Resistencia H-80, con una resistencia característica de $80 \mathrm{MPa}$ a los 28 días sobre probeta

(c) Consejo Superior de Investigaciones Científicas Licencia Creative Commons 3.0 España (by-nc) cilíndrica de $\phi 15 \times 30 \mathrm{~cm}$, se fabricó utilizando los mismos áridos calizos de machaqueo y el mismo cemento tipo I55A que se utilizan en la factoría de prefabricación para el hormigón normal H-45, de fabricación estándar para todos los prefabricados: vigas, pilotes y tubos (Fig. 8).

Para conseguir esta mayor resistencia se redujo el tamaño máximo del árido de $25 \mathrm{~mm}$ a $12 \mathrm{~mm}$, se aumentó la dosificación de cemento a $452 \mathrm{~kg} / \mathrm{m}^{3}$ y se añadió una cantidad de $23 \mathrm{~kg} / \mathrm{m}^{3}$ ( $5 \%$ del cemento) de microsílice densificada, de fabricación española, obtenida como subproducto de aleaciones de ferrosilicio.

La cantidad de agua se redujo a $1471 / \mathrm{m}^{3}$ y se aumentó la dosificación de superfluidificante a $141 / \mathrm{m}^{3}$. La cantidad de arena empleada fue de $889 \mathrm{~kg} / \mathrm{m}^{3}$, de grava fina $0 / 6 \mathrm{~mm}$, 
$333 \mathrm{~kg} / \mathrm{m}^{3}$ y de grava $2 / 12,634 \mathrm{~kg} / \mathrm{m}^{3}$. El peso específico del hormigón fresco fue de $2.492 \mathrm{~kg} / \mathrm{m}^{3}$ y el asiento, medido con el cono de Abrams, entre 12 y $16 \mathrm{~cm}$.

\section{Sistema de cálculo del HAR}

El cálculo de las vigas de HAR de los tableros se realizó utilizando las normas vigentes en España para acciones sísmicas y hormigón pretensado.

La vigente Instrucción española de hormigón pretensado EP-93, limita su ámbito de aplicación a hormigones de resistencia característica no superior a $60 \mathrm{MPa}(\mathrm{H}-60)$. El Eurocódigo 2, en fase de elaboración e implantación, también tiene el mismo límite. Por lo tanto, para poder utilizar el $\mathrm{H}-80$, se necesitaba extrapolar estas normas.

Se consultaron los siguientes documentos y normas sobre HAR:

\section{- Norma noruega N.S. 3473}

- Informe sobre el Estado del Arte del Hormigón de Alta Resistencia ACI-363-84.

- Proyecto europeo Brite Euram Project 5480. Proyecto y construcción económicos con Hormigón de Alta Resistencia.

De la información de esta documentación y de su análisis, se adoptaron las modificaciones siguientes a la EP-93:

- Módulo de elasticidad inicial: 32,0 GPa

- Módulo de elasticidad final: $37,0 \mathrm{GPa}$

- Cálculo del cortante: como hormigón H-60

\section{Control de las vigas}

\subsection{Descripción general}

Para conocer detalladamente el HAR y poder tener seguridad de su fabricación y uso correcto, se hicieron una serie de ensayos. Estos ensayos se realizaron primero sobre los materiales que forman el hormigón, para su correcta fabricación, después sobre hormigón fabricado, para poder conocer sus propiedades y su comportamiento, más tarde sobre el hormigón de fabricación de las vigas, para el control de la calidad y, por último, sobre las vigas y el puente construido (Fig. 9).

\subsection{Ensayos sobre los materiales del hormigón}

Se realizaron ensayos preliminares para la comparación entre los diferentes materiales que se podían utilizar para la fabricación del HAR. Se utilizaron para estos ensayos,

(c) Consejo Superior de Investigaciones Científicas Licencia Creative Commons 3.0 España (by-nc)

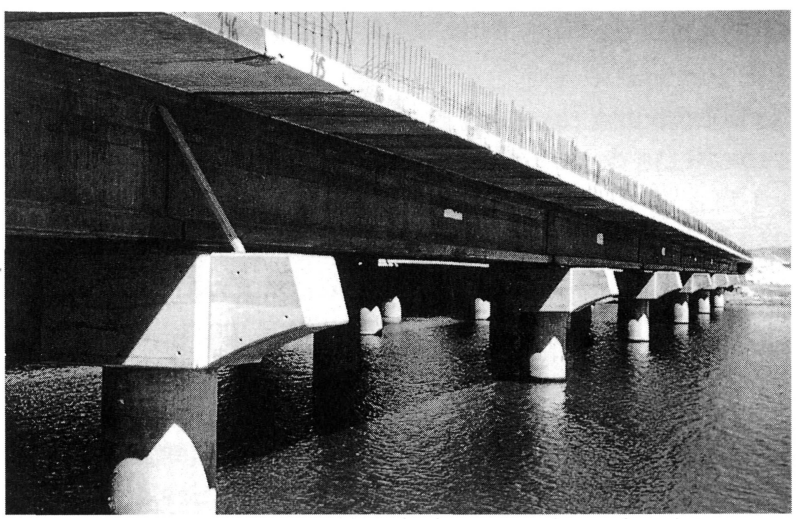

Fig. 9.- Fotografia del puente con los tableros terminados.

cementos, humos de sílice y superfluidificantes, de dos o tres fabricantes distintos por cada material.

Estos materiales se combinaron con áridos, arenas y gravas de varias naturalezas, calizas, silíceas y ofíticas y, en el caso de arenas, tanto de machaqueo como rodadas. Las diferencias que se obtuvieron en estos ensayos, tanto en resistencia como en docilidad y trabajabilidad, fueron muy pequeñas.

El cemento y el superfluidificante usados normalmente en la factoría y la microsílice, de fabricación española, dieron unos resultados algo mejores que el resto de los materiales alternativos utilizados.

La mejora que se obtenía al cambiar los áridos normalmente utilizados en la factoría por otros distintos, no compensaba los inconvenientes y problemas que producía el tener un mayor número de áridos en la planta de fabricación de hormigón, que requería aumentar el número de particiones para ellos.

Por ello, se decidió utilizar los mismos materiales, cemento, superfluidificante y áridos calizos de machaqueo, empleados en el resto de los elementos que se fabricaban en la factoría, con el único añadido del humo de sílice. Además, estos materiales eran bien conocidos a través de los ensayos sistemáticos de control de calidad y no precisaban otros ensayos, al conocerse su buen comportamiento en hormigones fabricados a lo largo de muchos años. Solamente fue preciso analizar la microsílice ( $89 \%$ de $\mathrm{SiO}_{2}$ ) y su actividad en comparación con el cemento, que fue del $107 \%$.

\subsection{Ensayos sobre el hormigón}

Se realizaron ensayos previos con una amasadora de laboratorio y después los ensayos característicos con la planta de hormigonado de la factoría, tal y como prescribe la norma EP-93. Se ensayaron dos dosificaciones: una, la que fue adoptada, con $475 \mathrm{~kg} / \mathrm{m}^{3}$ de conglomerante total $\left(452 \mathrm{~kg} / \mathrm{m}^{3}\right.$ de cemento y $23 \mathrm{~kg} / \mathrm{m}^{3}$ de humo de sílice) y otra, con un mayor contenido total de conglomerante, 
$500 \mathrm{~kg} / \mathrm{m}^{3}(465 \mathrm{~kg}$ de cemento y $35 \mathrm{~kg}$ de humo de sílice $-7,5 \%)$.

Se obtuvo una resistencia media en probeta cilíndrica de $\phi 15 \times 30 \mathrm{~cm}$ de $60 \mathrm{MPa}$ a 3 días, 69 a 7 días, 100 a 28 días y 108 a 90 días y una resistencia característica de 56, 64, 95 y 103 MPa respectivamente, para la dosificación que fue elegida. Para la otra dosificación, con mayor cantidad de conglomerante $\mathrm{y}$, por lo tanto, más cara, los valores obtenidos fueron superiores, pero no en más de $2 \mathrm{MPa}$.

Por lo tanto, la elección fue clara, ya que la elegida cumplía sobradamente con la resistencia característica de $80 \mathrm{MPa}$, definida en el proyecto para las vigas y era más económica.

Al mismo tiempo que se realizaron las amasadas en la planta de hormigonado, para los ensayos característicos, se comprobaron las posibles dificultades de distribución de hormigón en el encofrado y su vibrado, ya que el humo de sílice hace que el hormigón se vuelva pegajoso. Se resolvieron todos los problemas.

Una vez elegida y comprobada la dosificación del HAR para cumplir la resistencia a 28 días, era preciso determinar el tiempo de curado al vapor y espera, para cumplir otra condición del proyecto, realizar la transmisión del pretensado cuando la resistencia del hormigón alcanzase los $60 \mathrm{MPa}$

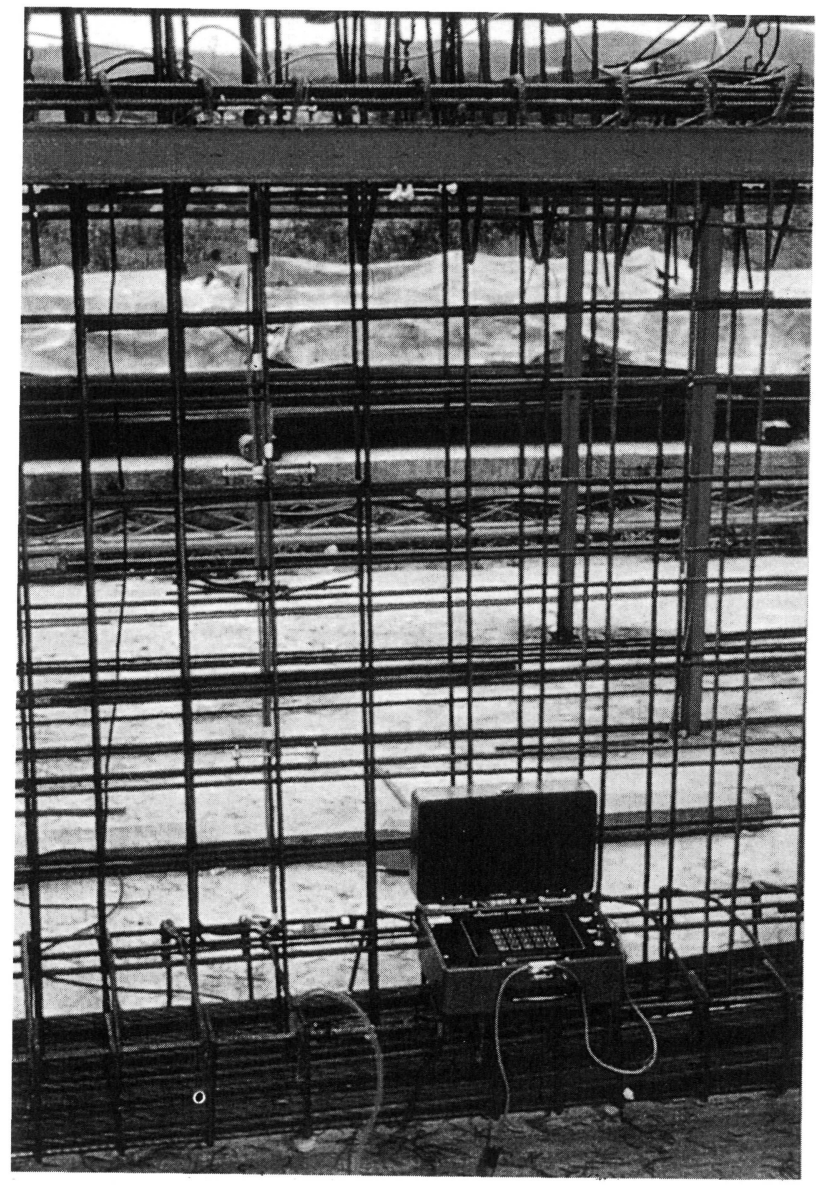

Se realizaron ensayos con probetas curadas a vapor, en condiciones similares a las de la bancada, durante 6, 12, 18 y 24 horas y se obtuvieron resistencias características de 55 , 61,64 y $68 \mathrm{MPa}$ respectivamente. De estos ensayos se dedujo un tiempo de curado al vapor de 12 horas como mínimo, antes de cortar los cables de la bancada.

Para conocer mejor las propiedades de HAR endurecido, se realizaron ensayos de resistencia a la tracción indirecta o brasileño, obteniéndose valores de 5,6 MPa a 7 días y de 6,7 MPa a 28 días. También se realizaron otros ensayos para determinar el módulo de elasticidad, y se obtuvieron resultados de $47,4 \mathrm{GPa}$ a 7 días y de $52,1 \mathrm{GPa}$ a 28 días.

Durante la fabricación se hicieron los ensayos de control, con una resistencia característica de $63 \mathrm{MPa}$ a 7 días y de $86 \mathrm{MPa}$ a 28 días.

\subsection{Ensayos de las vigas en la fabricación}

En el interior de dos de las vigas fabricadas, las correspondientes a un tablero, se dispusieron sensores para conocer mejor su comportamiento durante la fabricación y durante su vida posterior, una vez colocadas en el tablero del puente (Fig. 10).

Los sensores instalados fueron extensómetros de cuerda vibrante y termorresistencias.
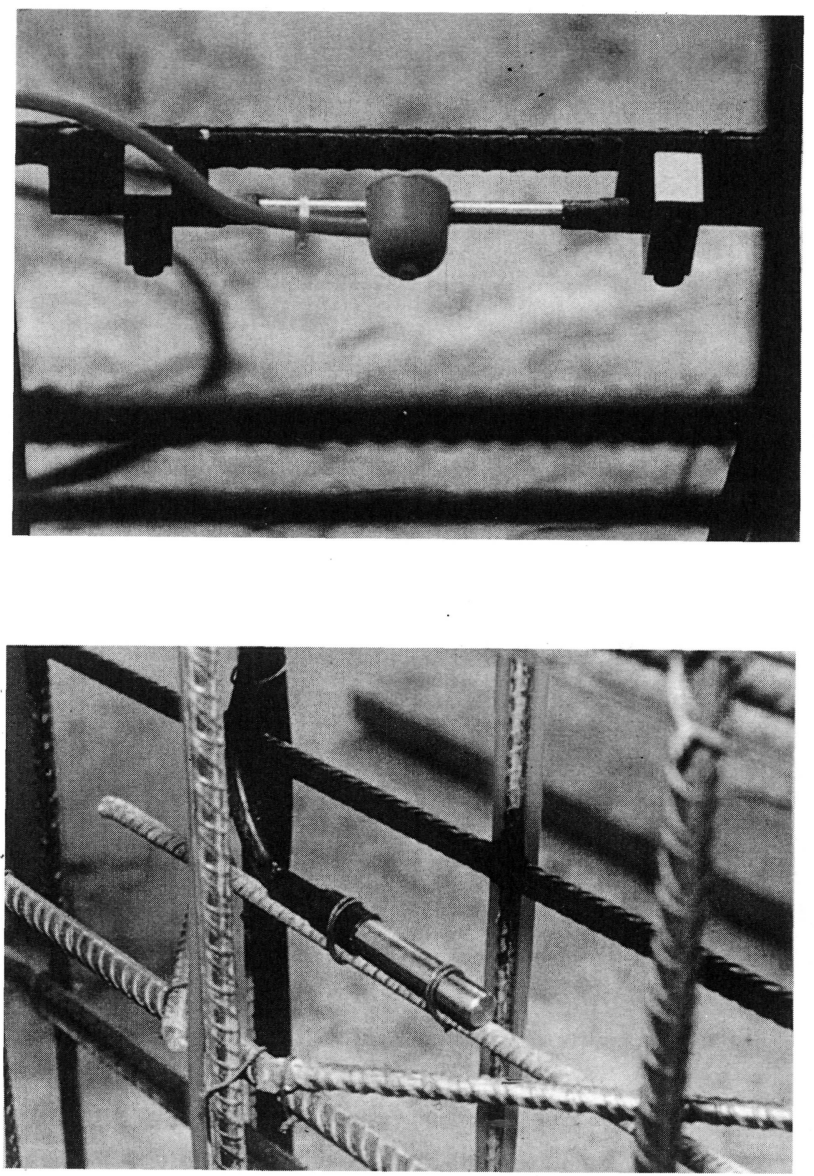

Fig. 10.- Fotografias de la instrumentación de una viga y de los sensores en detalle. 
Los extensómetros fueron colocados en las secciones centrales de las dos vigas, seis en cada una de ellas, dos en la fibra superior, dos en la fibra inferior y dos distribuidos en el alma.

Estos extensómetros fueron usados para medir las deformaciones de las fibras correspondientes de las vigas, al efectuar la transferencia a la viga de las fuerzas del pretensado, al cortar los cables anclados en la bancada, y la posterior evolución de estas deformaciones en el acopio de las vigas en la factoría, antes de ser transportadas a la obra.

En el momento de la transferencia del pretensado, se obtuvo $1,03 \mathrm{~mm} / \mathrm{m}$ de diferencia, entre las medias de las deformaciones de las fibras superior e inferior de las dos vigas. En este estado, después de la transferencia del pretensado, con la viga sometida a peso propio y a la fuerza del pretensado, la diferencia entre las tensiones de dichas dos fibras es de $36,9 \mathrm{MPa}$, lo que indica un módulo de elasticidad de 35,7 GPa.

Después de 28 días esta media de diferencia de deformaciones fue de $1,42 \mathrm{~mm} / \mathrm{m}$, para una diferencia de tensiones de $33,5 \mathrm{MPa}$, en un estado similar, sometidas las vigas a peso propio, pero considerando que ya se habían producido parte de las pérdidas totales por retracción y fluencia del hormigón. Esta diferencia representa un módulo de elasticidad de 23,7 GPa, lo que indica una relación de 1,5 para el efecto de la fluencia hasta esa fecha.

Las resistencias térmicas fueron colocadas en tres secciones de una sola de las dos vigas, cuatro en cada sección, en el centro de la cabeza superior, en el centro del alma, en el centro de la cabeza inferior y cerca de la pared en esta cabeza inferior. Las secciones elegidas fueron la central, una, a 2,40 m de ella y otra, a 4,80 m.

Las temperaturas se midieron con intervalos de tiempo de una hora, durante las primeras 30 horas y tres veces al día, durante los siguientes cinco días. La máxima temperatura

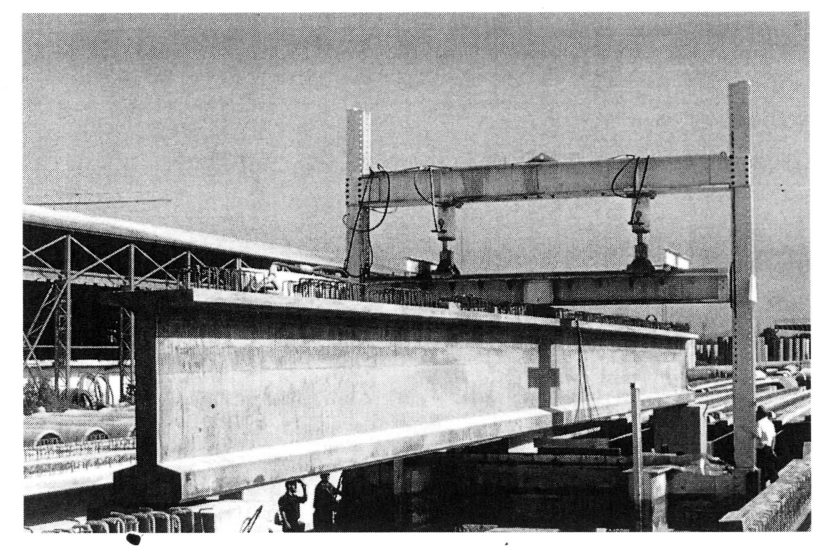

Fig. 11.- Fotografia de la viga durante la prueba de carga estática en la factoria.

(c) Consejo Superior de Investigaciones Científicas Licencia Creative Commons 3.0 España (by-nc) de $58^{\circ} \mathrm{C}$ se obtuvo en el centro de la cabeza superior, a las seis horas del comienzo del curado a vapor y ocho horas después del hormigonado de la viga. La viga disminuyó su temperatura, hasta la media de la temperatura ambiente, 4 días después del hormigonado.

\subsection{Ensayos sobre las vigas fabricadas}

Sobre las dos vigas instrumentadas se realizó una prueba de carga estática, en la propia factoría de prefabricación, antes de su envío a obra para ser colocadas en el puente (Fig. 11). La prueba se efectuó aplicando una carga de $150 \mathrm{t}$, aproximadamente en el centro de la luz de las vigas, por medio de dos gatos hidráulicos paralelos de $75 \mathrm{t}$ cada uno y de una viga metálica que se apoyaba sobre las vigas.

La prueba de carga se realizó individualmente para cada viga, 58 días después de su fabricación. La carga se aplicó en escalones aproximadamente iguales. En cada escalón se medía la flecha en el centro de la viga con un nivel de precisión y las deformaciones en las fibras, mediante la lectura de los extensómetros.

En una de las vigas la descarga se hizo también por escalones y en la otra de una sola vez, con medida de flechas y lectura de extensómetros en cada escalón y después de la descarga total, para verificar el grado de recuperación de flechasy deformaciones de las vigas, que fue prácticamente completo en ambas, poco después de la descarga.

Se pretendió alcanzar en la fibra inferior de las vigas, con la carga aplicada, la misma tensión de tracción que se debe producir en el tablero del puente construido (Fig. 12), si se les aplica el momento máximo de servicio para el que se han calculado, con todas las cargas permanentes y sobrecargas.

Estando la máxima carga de la prueba aplicada a las vigas, se inspeccionaron éstas con cuidado y detenimiento, buscando posibles fisuras, en especial en la cara inferior, no pudiéndose detectar ninguna fisura.

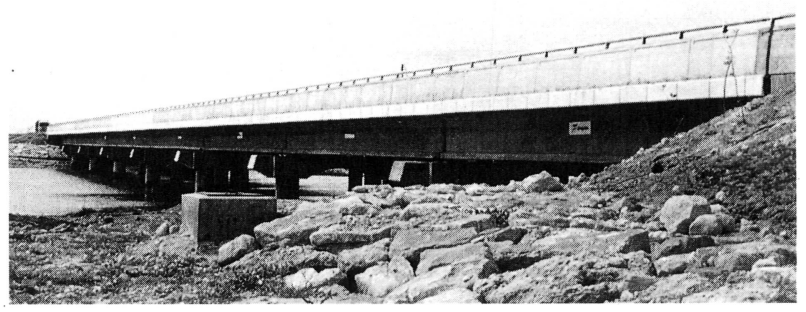

Fig. 12.- Fotografia del puente completamente terminado. 
A partir de las lecturas de los extensómetros y sus correspondientes deformaciones calculadas, se comprobó que la deformación de la sección central de la viga era prácticamente plana durante toda la prueba, como se puede esperar en una sección sin fisurar.

A partir de las flechas que se midieron y de las deformaciones de los extensómetros en las fibras estudiadas, se pudo calcular por dos sistemas el módulo de elasticidad del hormigón de las vigas, asumiendo que éstas habían permanecido con secciones sin fisurar durante la prueba.

Los valores obtenidos por los dos sistemas y para las dos vigas fueron muy similares. El valor medio del módulo de elasticidad obtenido fue de 51,0 GPa, valor muy similar también al obtenido en el laboratorio a los 28 días a partir de probetas, y que se ha descrito en un apartado anterior.

\section{Agradecimientos}

Los autores desean agradecer a la Dirección General de
Carreteras (MOPTMA), por financiar las obras de este puente descritas en el presente artículo, y en especial a las personas que participaron en este proyecto: D. Juan F. Lazcano, como Subdirector General de Construcción, D. José L. Elvira, como Subdirector General de Tecnología y D. José M. Delgado, como Director de Obra, por el apoyo, las directrices y la supervisión a lo largo de la investigación realizada para esta obra.

También desean agradecer el impulso y apoyo dado por el CEDEX y en especial a D. Carlos S. Oteo, como Director del Laboratorio de Geotecnia, al considerar este puente de vigas HAR, como una forma de ayudar a resolver, en su zona, la gran dificultad de realizar y apoyar en la marisma de Cádiz la carretera de la variante.

Por último, agradecer también la participación y colaboración de todas las personas que han intervenido, tanto en la investigación previa como en la construcción del puente, de las empresas INTECSA y GEOCISA y de los Servicios Técnicos y de la División PEYMA de DRAGADOS.

\title{
Publicación del Instituto Eduardo Torroja - CSIC
}

\author{
Número monográfico de INFORMES
}

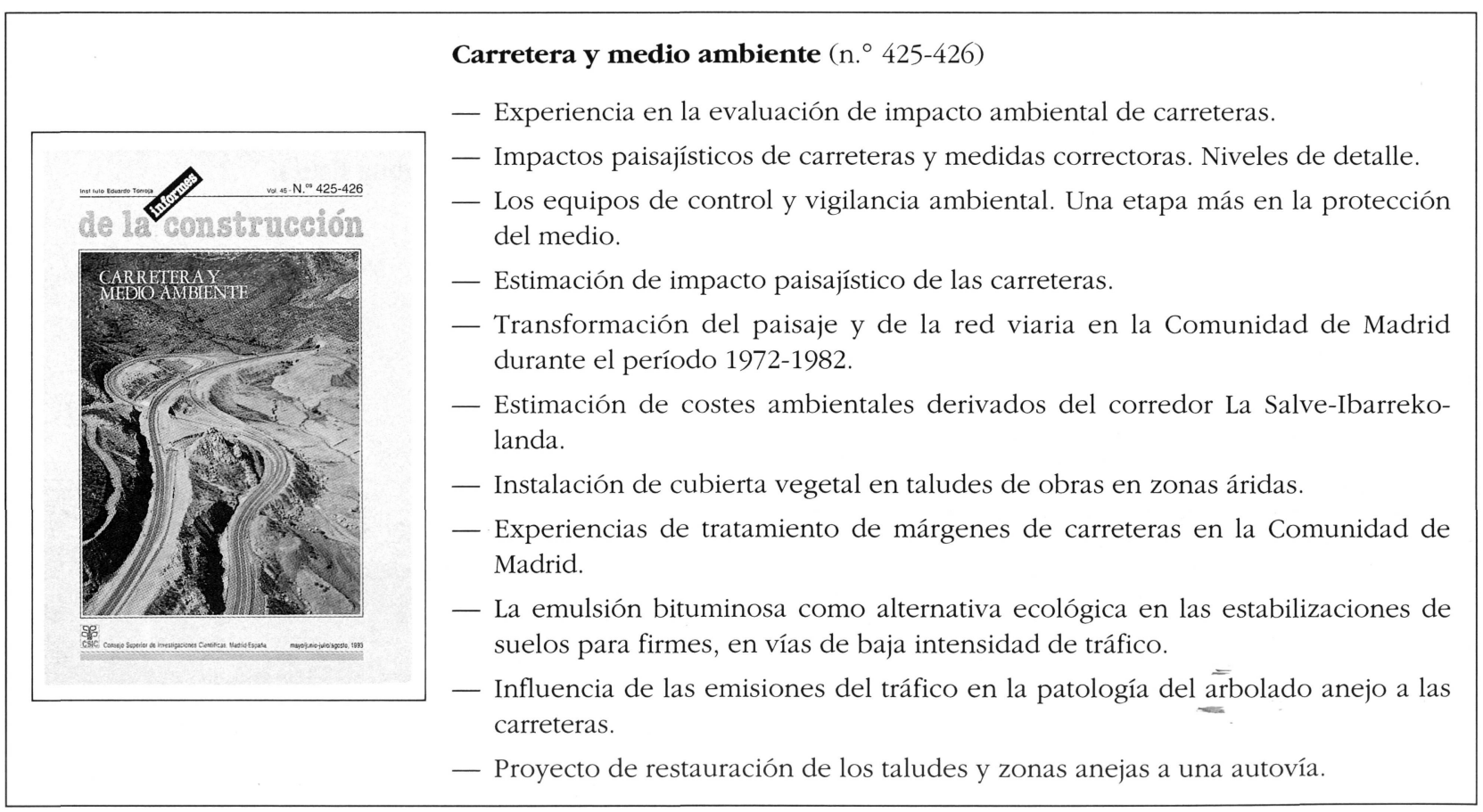

